\title{
Clinicopathological significance of the fragile histidine triad transcription protein expression in laryngeal carcinogenesis
}

\author{
KOICHI KITAMURA ${ }^{1}$, KEN HAYASHI $^{2,3}$, RYO KOBAYASHI $^{3}$, HIDEAKI ISHII $^{4}$, \\ JUN MATSUBAYASHI ${ }^{5}$, TETSUYA MATSUMOTO ${ }^{3}$ and MAMORU SUZUKI ${ }^{1}$
}

\author{
${ }^{1}$ Department of Otolaryngology, Tokyo Medical University, Tokyo; ${ }^{2}$ Department of Otolaryngology, Shinkawa Clinic, \\ Kanagawa; ${ }^{3}$ Department of Microbiology, Tokyo Medical University, Tokyo; ${ }^{4}$ Department of Pathology, Nippon Medical \\ School Musashi Kosugi Hospital, Kanagawa; ${ }^{5}$ Department of Pathology, Tokyo Medical University, Tokyo, Japan
}

Received September 5, 2007; Accepted December 21, 2007

\begin{abstract}
The fragile histidine triad (FHIT), frequently lost in many cancers, was identified as a candidate tumor suppressor gene at chromosome $3 p$ locus 14.2. Loss of the FHIT protein because of the alteration or loss of heterozygosity by genetic deletion occurs in a variety of epithelial tumors including head and neck cancer. However, the biological function of the FHIT protein is still unknown and its role in intrinsic cellular proliferation remains particularly controversial in preinvasive lesions and invasive tumors of the head and neck. To clarify the role of the FHIT protein in laryngeal squamous cell carcinoma (LSCC) and to examine whether the expression of FHIT could be a prognostic parameter for laryngeal carcinogenesis, we investigated the relationship between the expression of the FHIT protein, other tumor suppressor gene products (p53 and p16), the cellular proliferation marker (Ki-67) and the survival time of patients with LSCC. In our study, there were significant differences $(p<0.05)$ in the expression of FHIT between low grade dysplasia and LSCC. Additionally, survival time analysis showed a significant correlation between the reduction of FHIT expression and the length of disease-free survival $(\mathrm{p}<0.05)$ in patients with T1-T2 N0 laryngeal carcinoma. However, we did not confirm a relationship between the expression of FHIT, the other tumor suppressor gene products (p53 and p16) or the cellular proliferation marker (Ki-67). In conclusion, we provided evidence that the reduction of FHIT levels may be a useful prognostic indicator for the clinical outcome of laryngeal SCC. Our findings indicated that FHIT utilizes a pathway independent of p53 and is involved in abnormal cell proliferation via
\end{abstract}

Correspondence to: Dr Koichi Kitamura, Department of Otolaryngology, Tokyo Medical University, 6-7-1 Nishishinjuku, Shinjuku-ku, Tokyo 160-0023, Japan

E-mail:k-kita@wd5.so-net.ne.jp

Key words: fragile histidine triad, low grade dysplasia, laryngeal squamous cell carcinoma, laryngeal carcinogenesis the breakdown of $\mathrm{G}_{0}-\mathrm{G}_{1}$ arrest in the larynx and apoptosis during multistep carcinogenesis of the larynx.

\section{Introduction}

Head and neck squamous cell carcinoma (HNSCC) is a heterogeneous disease with complex molecular abnormalities. It is reported to arise from a premalignant progenitor followed by an outgrowth of clonal populations associated with cumulative genetic alterations and a phenotypic progression to invasive malignancy (1-3). These genetic alterations result in the inactivation of tumor suppressor genes and activation of proto-oncogenes by deletions, point mutations, promoter methylation and genetic amplification (1,4-7). Microsatellite marker analysis has allowed the delineation of a genetic progression model for HNSCC based on the frequency of these alterations in preinvasive lesions and invasive tumors $(1,3)$. Among them, the loss of chromosome region $3 p$ was reported to be a common early genetic event in squamous dysplasia and invasive HNSCC $(6,7)$. Recent molecular analysis showed that about $60-70 \%$ of HNSCC cases had a loss of heterozygosity in the chromosome arm $3 p(5)$. Fragile histidine triad (FHIT), located on the short arm of chromosome 3 at locus $3 p$ 14.2, is homologous to a family of genes characterized by histidine triad (8). FHIT has been identified as a tumor suppressor gene encompassing the most fragile site of the human genome, FRA3B (8). Loss of the FHIT protein because of the alteration or loss of heterozygosity by genetic deletion occurs in a variety of epithelial tumors including head and neck cancer (9-14). Reduction of FHIT expression is also reported to be more important for squamous cell carcinoma than adenocarcinoma (15). Moreover, the FHIT protein seems to act as a signaling molecule involved in the regulation of p53-independent apoptosis (16) and cell cycle control at the DNA check point (17). However, the biological function of the FHIT protein is still unknown and its role in intrinsic cellular proliferation remains particularly controversial in preinvasive lesions and invasive tumors of the head and neck (10). In this study, we investigated whether the expression of the FHIT protein was correlated with the expression of the other tumor suppressor genes, p53 and p16, during laryngeal squamous cell 
carcinoma (LSCC) by using immunohistochemistry. To define the relationship between the expression of FHIT protein and cellular proliferation, we also compared the expression of FHIT protein with the labeling index of Ki-67. The aim of this study was to examine whether the expression of FHIT could be used as a prognostic parameter for LSCC.

\section{Materials and methods}

Patients and tissue specimens. Seventy-nine patients with dysplasia or LSCC were studied. The patients underwent surgery at the Department of Otolaryngology, Tokyo Medical University and the Kosei Chuo Hospital, Tokyo, Japan between 2000 and 2004. The clinical and pathological characteristics are listed in Tables I and II. The specimens of dysplasia were classified into two grades according to the guidelines set by the General Rules of Clinical Studies on Head and Neck Cancer of the Japan Society for Head and Neck Cancer (18): 23 low grade and 7 high grade dysplasias. Biopsies from the primary laryngeal carcinoma were taken at the initial clinical presentation before treatment. Patients with T1-T2 N0 laryngeal cancer were treated with chemotherapy and radiation therapy, while patients with T3-T4 laryngeal cancer were treated with surgery or surgery after adjuvant chemotherapy. Survival data were available for all patients: the minimum length of follow-up care was 10 months. The T-stage of patients with LSCC was evaluated by the General Rules of Clinical Studies on Head and Neck Cancer of the Japan Society for Head and Neck Cancer (18). The nodal status was determined with histological evidence of metastatic LSCC.

Immunohistochemical staining. The samples were fixed in $10 \%$ buffered formalin and embedded in paraffin wax. Sections $(5 \mu \mathrm{m})$ were cut from wax blocks and mounted onto poly-L-lysin-coated glass slides (Matsumami, Tokyo, Japan). The slides were deparaffinized twice in xylene for 10 min, re-hydrated through graded ethanol to distilled water before incubation for $30 \mathrm{~min}$ with $0.3 \%$ hydrogen peroxidemethanol to inhibit endogenous peroxidase activity and heated in $0.01 \mathrm{M}$ citrate buffer $(\mathrm{pH}$ 6.0) in an autoclave for $10 \mathrm{~min}$ at $121^{\circ} \mathrm{C}$ for antigen retrieval. After being incubated for $20 \mathrm{~min}$ in a blocking solution containing $10 \%$ normal rabbit serum in $\mathrm{PBS}$, sections were incubated at $4{ }^{\circ} \mathrm{C}$ overnight in a humidified chamber with the primary antibodies.

Antibodies. The following antibodies were used: anti-human Ki-67 protein (clone MIB-1, mouse IgG1, diluted with PBS to $1: 100$, Dako, Glostrup, Denmark), anti-human p53 protein (clone DO-7, mouse IgG2b, diluted with PBS to 1:100, Dako), anti-human p16 protein (clone $6 \mathrm{H} 12$, mouse IgG2a, diluted with PBS to 1:100, Novocastra Laboratories Ltd., NewcastleUpon-Tyne, UK) and anti-human FHIT protein (ZR44, rabbit polyclonal antibody, diluted with PBS to 1:100, Zymed, South San Francisco, CA, USA). The sections were rinsed in PBS and stained by using the Envision kit (Dako). Sections were lightly counterstained with hematoxylin solution.

Evaluation of the immunohistochemical results. p53 and p16 staining was considered positive if $\geq 25 \%$ of the epithelial
Table I. Patient characteristics for laryngeal dysplasia.

\begin{tabular}{lccc}
\hline Characteristic & $\begin{array}{c}\text { No. of patients } \\
(\%)\end{array}$ & $\begin{array}{c}\text { FHIT (+) } \\
(\%)\end{array}$ & $\begin{array}{c}\text { FHIT (-) } \\
(\%)\end{array}$ \\
\hline Total & 30 & $17(56.7)$ & $13(43.3)$ \\
Age & & & \\
$\leq 60$ & $11(36.7)$ & $6(54.6)$ & $5(45.4)$ \\
$>60$ & $19(63.3)$ & $11(57.9)$ & $8(42.1)$ \\
Sex & & & \\
Male & $29(96.7)$ & $16(55.2)$ & $13(44.8)$ \\
Female & $1(3.3)$ & $1(100)$ & $0(0)$ \\
Degree of dysplasia & & & \\
$\quad$ Low grade & $23(76.7)$ & $15(65.2)$ & $8(34.8)$ \\
High grade & $7(23.3)$ & $2(28.6)$ & $5(71.4)$ \\
\hline
\end{tabular}

Table II. Patient characteristics for LSCC.

\begin{tabular}{lccc}
\hline Characteristic & $\begin{array}{c}\text { No. of patients } \\
(\%)\end{array}$ & $\begin{array}{c}\text { FHIT (+) } \\
(\%)\end{array}$ & $\begin{array}{c}\text { FHIT (-) } \\
(\%)\end{array}$ \\
\hline Total & 49 & $13(26.5)$ & $36(73.5)$ \\
Age & & & \\
$\leq 60$ & $12(24.5)$ & $2(16.7)$ & $10(83.3)$ \\
$>60$ & $37(75.5)$ & $11(29.7)$ & $26(70.3)$ \\
Sex & & & \\
Male & $47(95.9)$ & $11(23.4)$ & $36(76.6)$ \\
Female & $2(4.1)$ & $2(100)$ & $0(0)$ \\
T stage & & & \\
T1 and T2 & $33(67.3)$ & $10(30.3)$ & $23(69.7)$ \\
T3 and T4 & $16(32.7)$ & $3(18.8)$ & $13(81.2)$ \\
Nodal status & & & \\
N (-) & $39(80.0)$ & $11(28.2)$ & $28(71.8)$ \\
N (+) & $10(20.0)$ & $2(20.0)$ & $8(80.0)$ \\
\hline
\end{tabular}

cells showed nuclear staining and negative if $<25 \%$ of the epithelial cells showed nuclear staining (19). FHIT staining was also considered positive if $\geq 10 \%$ of the epithelial cells showed nuclear and/or cytoplasm staining $(14,20)$. The Ki-67 labeling index was calculated as the percentage of Ki-67positive cells that showed nuclear staining among 1,000 epithelial cells counted in $\geq 5$ fields of a specimen.

Statistical analysis. Survival curves for disease-free survival and overall survival time were estimated by the Kaplan-Meier method and the resulting curves were compared using the long-rank test. Disease-free survival time was calculated from the date of cancer treatment to relapse or death from cancerrelated causes. The statistical comparison of the two groups was performed using the Kruskal-Wallis test. All tests were two-tailed. A value of $\mathrm{p}<0.05$ was considered significant. 


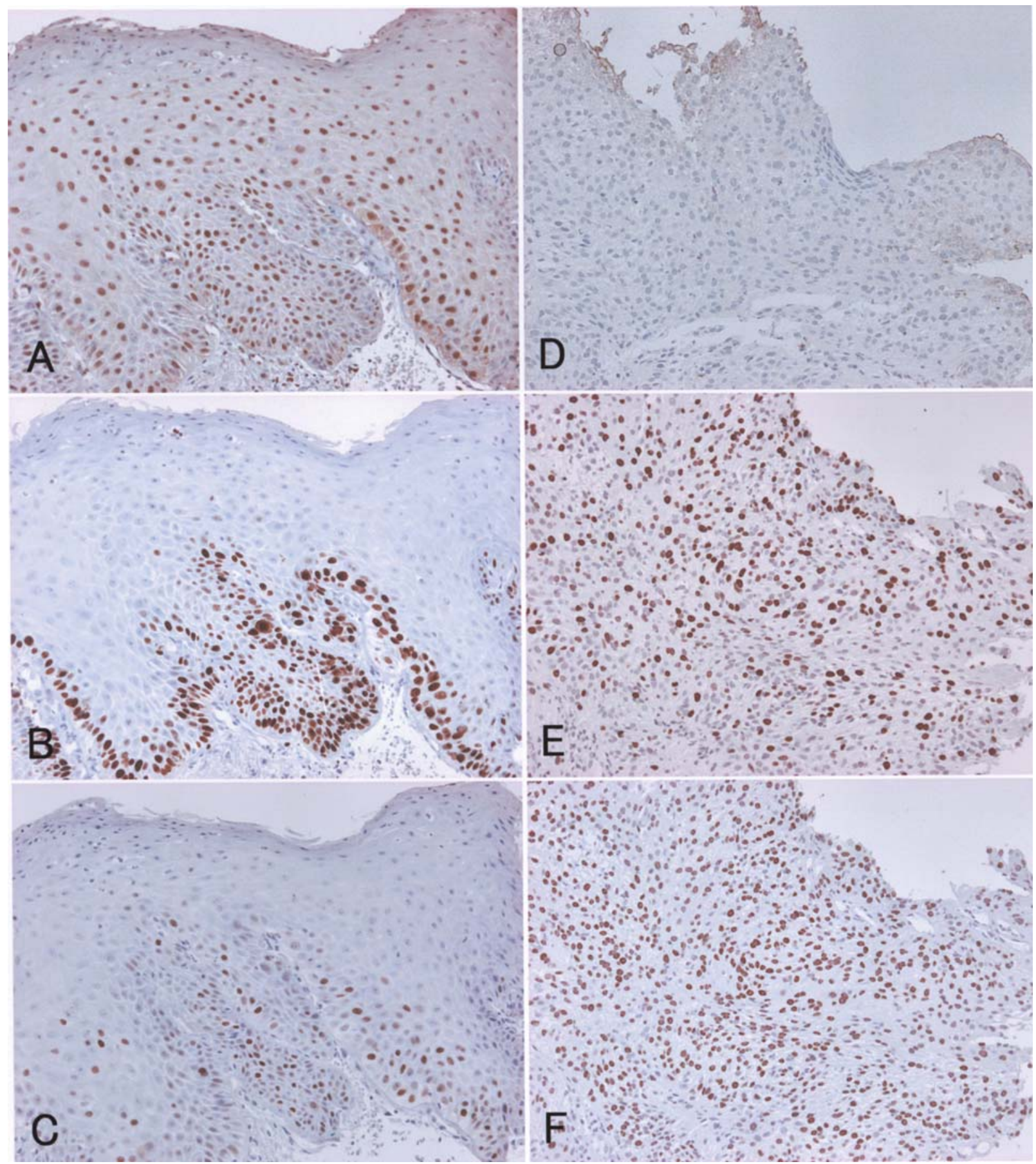

Figure 1. Immunohistochemistry for FHIT, Ki-67 and p53 proteins in the larynx. (A, B and C) Low grade dysplasia (same field). (D, E and F) SCC (same field). (A and D) FHIT protein staining. (B and E) Ki-67 protein staining. (C and F) p53 protein staining. The expression of FHIT protein was observed in nuclei and/or cytoplasm and Ki-67 and p53 proteins were expressed in the nuclei.

\section{Results}

FHIT expression in laryngeal carcinogenesis. Of the low grade dysplasias studied, $65.2 \%$ showed a strong FHIT expression in the nuclear and/or cytoplasmic sites of basal and mucosal layer cells (Figs. 1A and 2A). On the other hand, $73.5 \%$ of the LSCC cases clearly showed a reduced or absent FHIT staining (Figs. 1D and 2A). This difference in the protein expression between the LSCC and the low grade dysplasia cells was significant $(\mathrm{p}<0.01)$, but no significant difference was observed between LSCC and high grade dysplasia cells (Fig. 2A)

The relationship between FHIT expression and conventional prognostic factors. The results of FHIT immunostaining were compared with the expression of Ki-67. The distribution of Ki-67 positive cells in low and high grade dysplasias was only observed in the basal layer (Figs. 1B and 2B). The percentages of the Ki-67 labeling index in these groups were 25.8 and $28.0 \%$, respectively. In LSCC, $48.2 \%$ of the cases showed a 
A

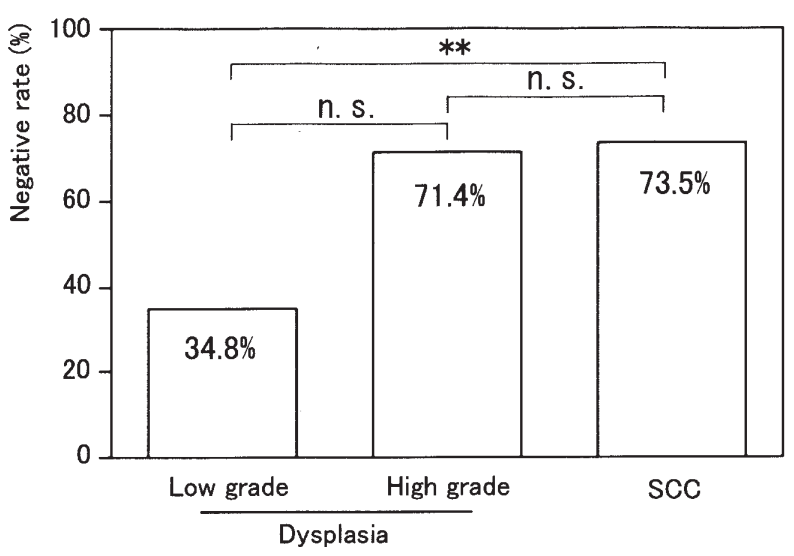

C

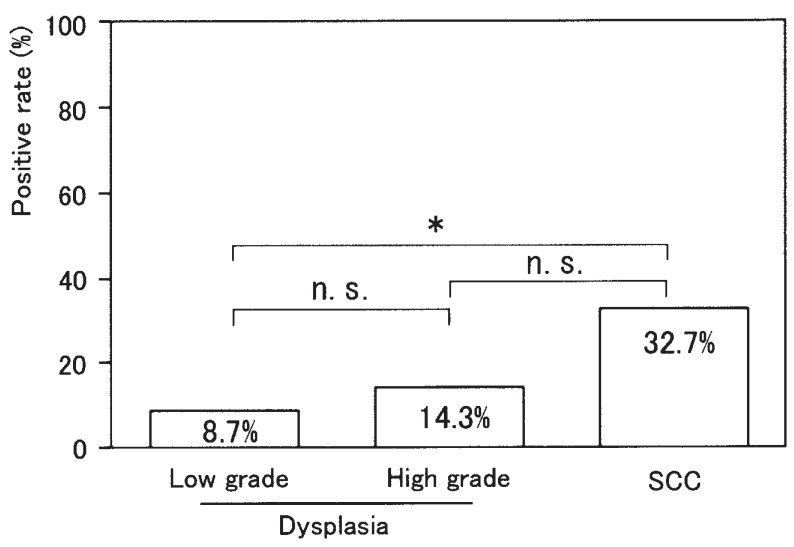

B

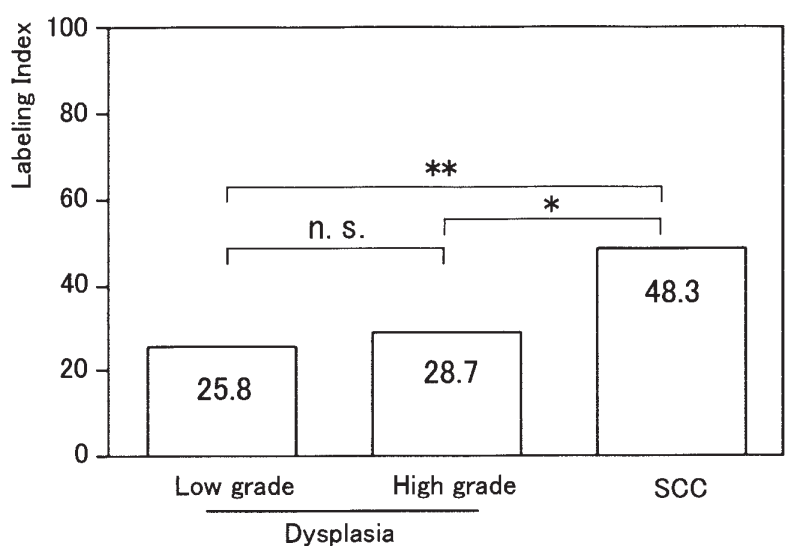

D

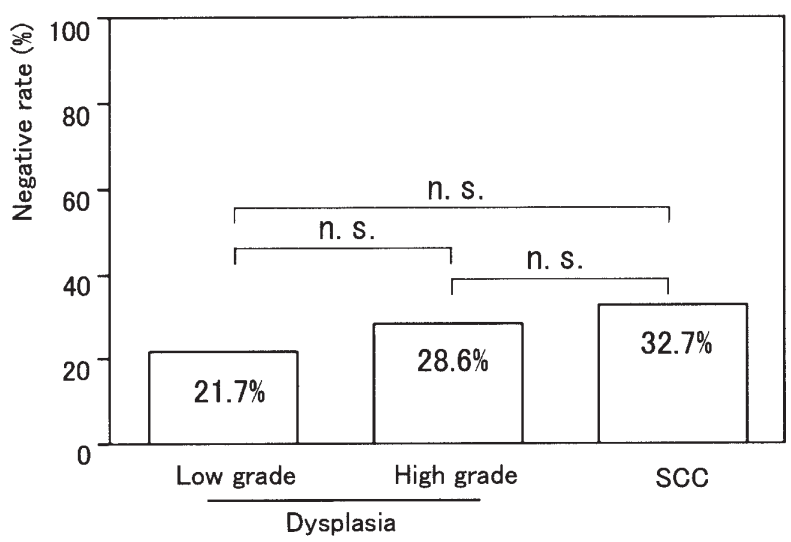

Figure 2. The percentage of FHIT, p53 and p16 positive specimens and the rate of Ki-67 labeling index in carcinogenesis of the larynx. (A) FHIT, (B) Ki-67, (C) $\mathrm{p} 53$ and (D) p16. ${ }^{*} \mathrm{p}<0.05 ;{ }^{* *} \mathrm{p}<0.01$ and n.s. $=$ no significant difference.

strong Ki-67 expression in whole cancer cells (Figs. 1E and 2B). There were significant differences $(\mathrm{p}<0.01$ and 0.05 , respectively) in the Ki-67 protein expression between LSCC and low/high grade dysplasias (Fig. 2B). The expression of p53 or p16 proteins in the LSCC and dysplasias was also examined. A p53 overexpression was observed in $32.7 \%$ of the LSCC cases and in $8.7 \%$ of the low grade dysplasias (Figs. 1C and $2 \mathrm{C})$. There was a significant difference in the $\mathrm{p} 53$ protein expression between these groups $(\mathrm{p}<0.05)$. On the other hand, no differences were evident in the 16 protein expression between low/high grade dysplasias and LSCC (Fig. 2D).

The relationship between FHIT expression and clinical parameters in multivariate analysis. We subsequently analyzed the relationship between FHIT expression and the length of survival. Fig. 3A shows a comparison of the Kaplan-Meier overall survival curve between patients with FHIT-positive and -negative tumors. There were no significant differences between the FHIT expression and the length of survival (overall survival, $\mathrm{p}=0.115$; $\mathrm{T} 1-\mathrm{T} 2$ survival, $\mathrm{p}=0.337$ and $\mathrm{T} 3-\mathrm{T} 4$ survival, $\mathrm{p}=0.190$ ), although patients whose tumors were negative for FHIT expression tended to have a shorter survival time than patients positive for FHIT expression. When disease-free survival time ( $\mathrm{p}=0.194)$ was analyzed (Fig. 3B), patients with T1-T2 laryngeal carcinoma who were FHIT- negative demonstrated a significantly worse prognosis than patients who were FHIT-positive $(\mathrm{p}=0.039)$. However, there were no significant differences between the FHIT expression and the length of disease-free survival in patients with T3-T4 laryngeal cancer $(\mathrm{p}=0.546)$. We also investigated other clinical parameters in relation to survival time. The FHIT expression was not correlated to any other clinical parameters and no statistical differences were found between patients with FHITnegative and -positive tumors using a two-tailed t-test. The following variables were included for multivariate analysis: gender (male versus female), age ( $\leq 60$ versus $>60$ ), tumor stage (T1 and 2 versus T3 and 4) and nodal status [N (-) versus $\mathrm{N}(+)$ ], as well as FHIT expression (FHIT-negative versus -positive).

\section{Discussion}

The FHIT gene, which belongs to the histidine triad superfamily, has been mapped to p14.2 of human chromosome 3 (8). It encodes a cytoplasmic protein that has diagnosine triphosphate activity (21). The most common fragile site of the genome, FRA3B, maps within the FHIT gene (8). This fragile site makes FHIT susceptible to rearrangements induced by a variety of carcinogens. Additionally, the FHIT gene is thought to have a tumor suppressor activity. Although the mechanism 

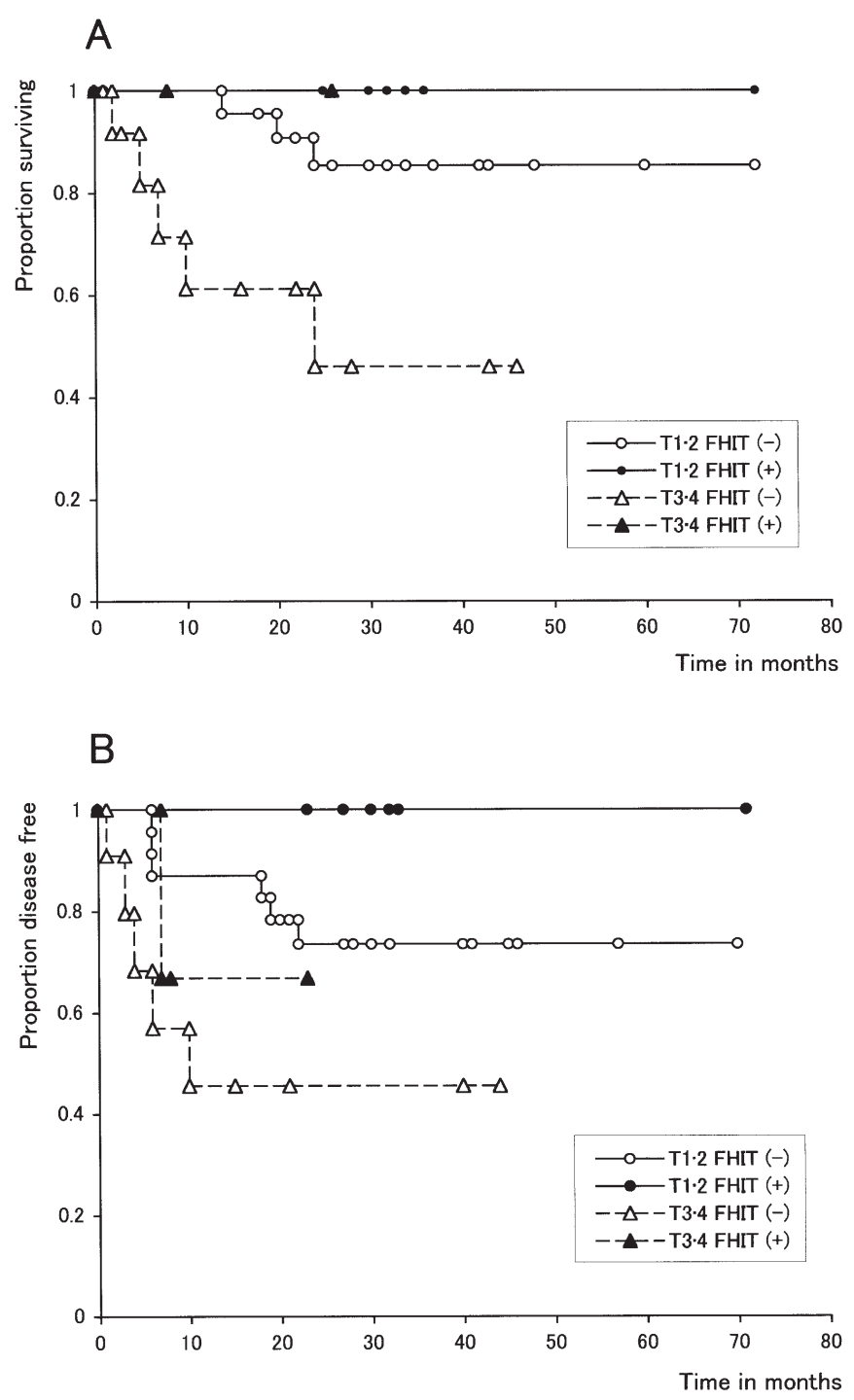

Figure 3. (A) Overall survival curves of patients with LSCC according to FHIT expression. (B) Disease-free survival curves of patients with LSCC according to the FHIT expression.

for inactivating the FHIT gene has not been fully shown, the genetic and epigenetic inactivation of the FHIT gene has been demonstrated in multiple tumor types (22-26). Furthermore, Tomizawa et al (27) have reported that alteration of the FHIT locus detected by DNA and reverse transcription-PCR analysis correlated well with the loss of FHIT protein expression in lung cancer. Segawa et al (28) found an abnormal FHIT protein expression in endometrial carcinoma. These findings indicated that FHIT gene alteration can simply be detected through the protein analysis of tumor specimens. Gatalica et al (29) showed that loss of FHIT expression was evident in preneoplastic lesions such as hyperplasia, and that the loss of expression increased during multistep carcinogenesis. In this study, a reduced FHIT expression was observed in $34.8 \%$ of low grade dysplasia and $71.4 \%$ of high grade dysplasia of the larynx, and $73.5 \%$ of laryngeal cancer specimens. There were significant differences between low grade dysplasia and LSCC. The expression of FHIT was suddenly reduced in high grade dysplasia, although there were no significant differences between low and high grade dysplasia. On the contrary, no association was found between FHIT expression and patient outcome measures including tumor grade and stage, and the nodal status of LSCC. These points show that FHIT probably plays an important role in the early phase of laryngeal carcinogenesis rather than in the progression to invasive and metastatic disease. However, survival time analysis demonstrated a significant correlation between the reduction of FHIT expression and the length of disease-free survival $(\mathrm{p}<0.05)$ in patients with T1-T2 N0 laryngeal carcinoma. These results suggest that FHIT may be a potential prognostic marker as well as a therapeutic target for early stage laryngeal cancer.

Our study examined whether the expression of FHIT was correlated with other markers of poor prognosis (p53 and p16) and cellular proliferation (Ki-67 labeling index), as well as known clinical and pathological variables. p53 alterations have mainly been found in the process of HNSCC during the late aggressive stage of HNSCC development (10). Our results also found that there were significant differences between a low grade dysplasia of the larynx $(8.7 \%)$ and laryngeal carcinoma $(32.7 \%)$ with regards to p53 accumulation, whereas no significant differences were detected between the reduction of the FHIT protein and p53 accumulation in dysplasia and laryngeal cancer. This result shows that the biological pathway of FHIT is independent from the pathway of p53 in laryngeal carcinogenesis. On the other hand, there were significant differences between low grade laryngeal dysplasia (25.8\%) and laryngeal carcinoma (48.3\%) in the Ki-67 labeling index, whereas no significant differences between the reduction of the FHIT protein and the Ki-67 labeling index were found in dysplasia and LSCC. This result indicates that FHIT may function in the $\mathrm{G}_{0}$ phase, because Ki-67 is a measure of the cellular proliferation index but not the $\mathrm{G}_{0}$ phase.

Sard et al (17) reported that FHIT was associated with $\mathrm{G}_{0}-\mathrm{G}_{1}$ arrest of lung cancer cells and the induction of apoptosis. Basically, the reduction of the FHIT levels may lead to the breakdown of $\mathrm{G}_{0}-\mathrm{G}_{1}$ arrest and apoptosis during laryngeal carcinogenesis and cancer cell proliferation. The $\mathrm{p} 16$ protein is reported to be part of the retinoblastoma pathway that regulates cell cycle progression from the $G_{1}$ to $S$ phase (30). The inactivation of p16 may lead to an imbalance in the cell cycle regulation, thereby inducing unrestricted proliferation and carcinogenesis including head and neck squamous cell cancer. Papadimitrakopoulou et al (31) found that $47 \%$ of patients with oral premalignant lesions showed a lack of p16 protein expression as detected by immunohistochemistry and they showed that p16 was inactivated during the early stages of head and neck carcinogenesis. However, our results showed the negative rate of p16 was $21.7 \%$ for low grade dysplasia, $28.7 \%$ for high grade dysplasia and $32.7 \%$ for LSCC, and there were no significant differences. Moreover, no correlation between the reduction of FHIT and p16 was found. We therefore suggest that the reduction of p16 protein levels and the breakdown of the $G_{1}$ to $S$ phase transition are not related to laryngeal carcinogenesis.

In conclusion, we provide evidence that the reduction of FHIT levels may be a useful prognostic indicator for the clinical outcome of laryngeal SCC. Our findings indicate that FHIT utilizes a pathway independent of p53, and induces abnormal cell proliferation through the breakdown of $G_{0}-G_{1}$ 
arrest in the larynx and apoptosis during multistep carcinogenesis of the larynx.

\section{References}

1. Califano J, van der Riet P, Westra W, et al: Genetic progression model for head and neck cancer: implications for field cancerization. Cancer Res 56: 2488-2492, 1996.

2. Nawroz H, van der Riet P, Hruban RH, Koch W, Ruppert JM and Sidransky D: Allelotype of head and neck squamous cell carcinoma. Cancer Res 54: 1152-1155, 1994.

3. Califano J, Westra WH, Meininger G, Corio R, Koch WM and Sidransky D: Genetic progression and clonal relationship of recurrent premalignant head and neck lesions. Clin Cancer Res 6: 347-352, 2000 .

4. van der Riet P, Nawroz H, Hruban RH, et al: Frequent loss of chromosome 9p21-22 early in head and neck cancer progression. Cancer Res 54: 1156-1158, 1994.

5. Garnis C, Baldwin C, Zhang L, Rosin MP and Lam WL: Use of complete coverage array comparative genomic hybridization to define copy number alterations on chromosome $3 \mathrm{p}$ in oral squamous cell carcinomas. Cancer Res 63: 8582-8585, 2003.

6. Hogg RP, Honorio S, Martinez A, et al: Frequent 3p allele loss and epigenetic inactivation of the RASSF1A tumour suppressor gene from region 3 p21.3 in head and neck squamous cell carcinoma. Eur J Cancer 38: 1585-1592, 2002.

7. Rowley H, Jones A, Spandidos D and Field J: Definition of a tumor suppressor gene locus on the short arm of chromosome 3 in squamous cell carcinoma of the head and neck by means of microsatellite markers. Arch Otolaryngol Head Neck Surg 122: 497-501, 1996.

8. Ohta M, Inoue H, Cotticelli MG, et al: The FHIT gene, spanning the chromosome 3 p14.2 fragile site and renal carcinomaassociated $\mathrm{t}(3 ; 8)$ breakpoint, is abnormal in digestive tract cancers. Cell 84: 587-597, 1996.

9. Tanimoto K, Hayashi S, Tsuchiya E, et al: Abnormalities of the FHIT gene in human oral carcinogenesis. Br J Cancer 82: 838-843, 2000.

10. Virgilio L, Shuster M, Gollin SM, et al: FHIT gene alterations in head and neck squamous cell carcinomas. Proc Natl Acad Sci USA 93: 9770-9775, 1996.

11. Kisielewski AE, Xiao GH, Liu SC, et al: Analysis of the FHIT gene and its product in squamous cell carcinomas of the head and neck. Oncogene 17: 83-91, 1998 .

12. Chang KW, Kao SY, Tzeng RJ, et al: Multiple molecular alterations of FHIT in betel-associated oral carcinoma. J Pathol 196: 300-306, 2002.

13. van Heerden WF, Swart TJ, van Heerden MB, et al: Immunohistochemical evaluation of Fhit protein expression in oral squamous cell carcinomas. J Oral Pathol Med 28: 433-437, 1999.

14. Mineta H, Miura K, Takebayashi S, et al: Low expression of fragile histidine triad gene correlates with high proliferation in head and neck squamous cell carcinoma. Oral Oncol 39: 56-63, 2003.

15. Mascaux C, Martin B, Verdebout JM, Meert AP, Ninane V and Sculier JP: Fragile histidine triad protein expression in nonsmall cell lung cancer and correlation with Ki-67 and with p53. Eur Respir J 21: 753-758, 2003.
16. Kitamura A, Yashima K, Okamoto E, et al: Reduced Fhit expression occurs in the early stage of esophageal tumorigenesis: no correlation with p53 expression and apoptosis. Oncology 61: 205-211, 2001.

17. Sard L, Accornero P, Tornielli S, et al: The tumor-suppressor gene FHIT is involved in the regulation of apoptosis and in cell cycle control. Proc Natl Acad Sci USA 96: 8489-8492, 1999.

18. General Rules of Clinical Studies on Head and Neck Cancer. Jpn Soc Head Neck Cancer 4: 50-51, 2005.

19. Jeannon JP, Soames JV, Aston V, Stafford FW and Wilson JA: Molecular markers in dysplasia of the larynx: expression of cyclin-dependent kinase inhibitors p21, p27 and p53 tumour suppressor gene in predicting cancer risk. Clin Otolaryngol Allied Sci 29: 698-704, 2004.

20. Lee JI, Soria JC, Hassan K, et al: Loss of Fhit expression is a predictor of poor outcome in tongue cancer. Cancer Res 61: 837-841, 2001.

21. Barnes LD, Garrison PN, Siprashvili Z, et al: Fhit, a putative tumor suppressor in humans, is a dinucleoside 5',5'"'-P1,P3triphosphate hydrolase. Biochemistry 35: 11529-11535, 1996.

22. Wali A, Srinivasan R, Shabnam MS, Majumdar S, Joshi K and Behera D: Loss of fragile histidine triad gene expression in advanced lung cancer is consequent to allelic loss at 3p14 locus and promoter methylation. Mol Cancer Res 4: 93-99, 2006.

23. Zochbauer-Muller S, Fong KM, Maitra A, et al: 5' CpG island methylation of the FHIT gene is correlated with loss of gene expression in lung and breast cancer. Cancer Res 61: 3581-3585, 2001.

24. Tanaka H, Shimada Y, Harada H, et al: Methylation of the 5' CpG island of the FHIT gene is closely associated with transcriptional inactivation in esophageal squamous cell carcinomas. Cancer Res 58: 3429-3434, 1998

25. Hayashi S, Tanimoto K, Hajiro-Nakanishi K, et al: Abnormal FHIT transcripts in human breast carcinomas: a clinicopathological and epidemiological analysis of 61 Japanese cases. Cancer Res 57: 1981-1985, 1997.

26. Sozzi G, Veronese ML, Negrini M, et al: The FHIT gene 3p14.2 is abnormal in lung cancer. Cell 85: 17-26, 1996.

27. Tomizawa Y, Nakajima T, Kohno T, Saito R, Yamaguchi N and Yokota J: Clinicopathological significance of Fhit protein expression in stage I non-small cell lung carcinoma. Cancer Res 58: 5478-5483, 1998

28. Segawa T, Sasagawa T, Saijoh K and Inoue M: Clinicopathological significance of fragile histidine triad transcription protein expression in endometrial carcinomas. Clin Cancer Res 6: 2341-2348, 2000.

29. Gatalica Z, Lele SM, Rampy BA and Norris BA: The expression of Fhit protein is related inversely to disease progression in patients with breast carcinoma. Cancer 88: 1378-1383, 2000.

30. Sherr CJ and Roberts JM: CDK inhibitors: positive and negative regulators of G1-phase progression. Genes Dev 13: 1501-1512, 1999.

31. Papadimitrakopoulou V, Izzo J, Lippman SM, et al: Frequent inactivation of p16INK4a in oral premalignant lesions. Oncogene 14: 1799-1803, 1997. 